\title{
Sources of Resistance to Diaprepes Weevil in Subfamily Aurantiodeae, Rutaceae
}

\author{
Kim D. Bowman' 1 Jeffrey P. Shapiro ${ }^{2}$, and Stephen L. Lapointe ${ }^{3}$ \\ U.S. Department of Agriculture, Agricultural Research Service, U.S. Horti- \\ cultural Research Laboratory, 2001 South Rock Road, Fort Pierce, FL 34945
}

Additional index words. insect injury, Diaprepes abbreviatus, citrus, rootstock

\begin{abstract}
Commercially used citrus rootstocks can all be seriously damaged by larvae of the sugar cane root weevil, Diaprepes abbreviatus (L.). Six greenhouse challenge experiments were used to compare the resistance of 24 selections of citrus rootstocks and related species to this weevil by measuring root damage, and larval growth and survival. The commercial rootstocks tested were found to be susceptible, while at least seven other species within the subfamily Aurantiodeae were observed to be significantly more resistant. The species Balsamocitrus dawei Stapf. was most resistant to weevil larvae, exhibited less root damage than commonly used rootstock cultivars, and significantly depressed larval growth and survival. The species Glycosmis pentaphylla (Retz.) Correa, Microcitrus australis (Planch.) Swing., Eremocitrus glauca (Lindl.) Swing., Severinia buxifolia (Poir.) Tenore, Triphasia trifolia (Burm. f.) P. Wils., and Citrus hystrix DC. suffered as much damage from the weevil as common rootstock cultivars but significantly depressed growth of larvae feeding on them. One new hybrid rootstock, HRS-801, also significantly depressed $D$. abbreviatus larval growth, but this effect has not yet been verified as having significance in a long-term or field situation. Several strategies are discussed for developing citrus rootstocks resistant to $D$. abbreviatus.
\end{abstract}

The sugar cane root weevil is a devastating pest of citrus and other crops in many parts of Florida and the Caribbean (Beavers et al., 1979; Schroeder and Beavers, 1977). Since its first discovery in Florida in 1964, D. abbreviatus has spread from a small area near Apopka (Orange County) to encompass 20 counties from central to south Florida. The adult weevil feeds on the foliage, but the main damage to the citrus tree is caused by larval feeding on roots. Some chemical and biological control agents are available, but no combination of these is both affordable and effective (Anonymous, 1994; McCoy et al., 1996;

Received for publication 10 Dec. 1998. Accepted for publication 29 Sept. 1999. Some of the seeds for this work were provided by the USDA Clonal Germplasm Repository for Citrus and Dates, Riverside, Calif. The described research was funded, in part, by grant No. 951-6 from the Florida Citrus Production Research Advisory Council. Mention of a trademark, warranty, proprietary product, or vendor does not imply an approval to the exclusion of other products or vendors that also may be suitable. We thank Tom Moyer, Hunter Smith, Charles Spriggs, Diane Weeks, and Connie Chiang for technical support on this project, and Victor Chew for assistance with statistical analysis. The cost of publishing this paper was defrayed in part by the payment of page charges. Under postal regulations, this paper therefore must be hereby marked advertisement solely to indicate this fact.

${ }^{1}$ Research Geneticist. To whom requests for reprints should be addressed. E-mail address: kbowman@ ushrl.ars.usda.gov

${ }^{2}$ Research Entomologist. Current address: USDA, ARS, CMAVE, 1700 SW 23rd Drive, Gainesville, FL 32608 .

${ }^{3}$ Research Entomologist.
Schroeder, 1988). Although many different citrus rootstocks are available, previous efforts to identify cultivars with a significant level of field resistance to $D$. abbreviatus have been unsuccessful (Beavers and Hutchison, 1985; Grosser and McCoy, 1996; Norman et al., 1974; Shapiro and Gottwald, 1995). Recently, we reported that one citrus relative, Glycosmis pentaphylla, was resistant to Diaprepes weevil in greenhouse testing and diet feeding assays (Shapiro et al., 1997).

The objectives of this study were to survey the subfamily Aurantiodeae and some untested rootstock cultivars for resistance to D. abbreviatus. Testing was conducted in six separate greenhouse experiments using the common rootstock 'Carrizo' citrange as a standard, susceptible genotype for comparison. Root loss, larval survival, and larval growth were measured.

\section{Materials and Methods}

Seeds of selected citrus rootstocks and relatives were harvested from fruit, dried, and stored at $4{ }^{\circ} \mathrm{C}$ until use. A special effort was made to include citrus relatives that had not been tested previously for resistance to Diaprepes root weevil (Table 1). Common rootstocks used for $\approx 85 \%$ of the commercial acreage in Florida and several promising new rootstock cultivars were also tested (Table 2). Seeds of B. dawei, C. hystrix, Clymenia polyandra (Tan.) Swing., E. glauca, Fortunella hindsii (Champ.) Swing., G. pentaphylla (Expt. 6) and M. australis were provided by the U.S. Dept. of Agriculture (USDA) National Clonal Germplasm Reposi- tory for Citrus and Dates (Riverside, Calif.) Seeds of Afraegle paniculata (Schum.) Engl., G. pentaphylla (Expts. 3 and 5), Murraya paniculata (L.) Jack, S. buxifolia, and T. trifolia were collected from the Florida Citrus Arboretum, Winter Haven. All other seeds were obtained from the USDA Whitmore Foundation Farm, Groveland, Fla. Seeds were planted in a peat/perlite/vermiculite potting mix (Pro-Mix BX: Premier Horticulture, Red Hill, $\mathrm{Pa}$.) at a rate of one seed per cell (140 $\mathrm{cm}^{3}$ volume) in multi-cell trays (Multi-Pots; Can-Am Containers, Springhill, N.S.). Citrus hystrix plants were propagated as cuttings from two uniform seedlings to minimize the effects of seedling variability. For all other genotypes, uniform healthy seedlings were selected for testing from larger populations. Individual seedlings were transplanted to 3.7-L containers when they were 3-9 months old and maintained on elevated benches in a greenhouse with an average diurnal temperature cycle of $35^{\circ} \mathrm{C}$ maximum and $23^{\circ} \mathrm{C}$ minimum in the summer, and $32^{\circ} \mathrm{C}$ and $20^{\circ} \mathrm{C}$ in the winter. For Expts. $1-5$, the same potting mix was used for transplanting to 3.7-L containers as for seed starting. For Expt. 6, plants were transplanted into 3.7-L containers using a potting mix lacking perlite (Metro Mix 500; Scotts Sierra Horticultural Products Co., Marysville, Ohio) to make it easier to separate the larvae from the potting mix at the end of the experiment. At the time of transplanting, $0.5 \mathrm{~g}$ Kocide 101 (cupric hydroxide; Griffin Corp., Valdosta, Ga.) and $1 \mathrm{~g}$ Sequestrene $138 \mathrm{Fe}$ (chelated iron; CibaGeigy Corp., Greensboro, N.C.) were applied to the containers to provide copper and iron to the growing plants. No supplemental light was supplied. The natural photoperiod fluctuated from $13.5 \mathrm{~h}$ in summer (using sunrise to sunset) to $10.25 \mathrm{~h}$ in December. Maximum photosynthetic photon flux $(P P F)$ in the greenhouse was $800 \mu \mathrm{mol} \cdot \mathrm{s}^{-1} \cdot \mathrm{m}^{-2}$. During initial growth, irrigation alternated between nonamended well water and a water soluble fertilizer mix, $15 \mathrm{~N}-7 \mathrm{P}-14 \mathrm{~K}$ (Peters Fertilizer Products; W.R. Grace, Fogelsville, $\mathrm{Pa}$.) applied with a proportioner at a rate of $380 \mathrm{mg} \cdot \mathrm{L}^{-1} \mathrm{~N}$. During the later stages of growth and the period of infestation, plants were watered with a dilute fertilizer mix at every irrigation using a water soluble $20 \mathrm{~N}-$ $4.3 \mathrm{P}-16.6 \mathrm{~K}$ at a rate of $150 \mathrm{mg} \cdot \mathrm{L}^{-1} \mathrm{~N}$.

When plants were $0.75-1.5 \mathrm{~m}$ tall and $7-$ 18 months old (27 months old for Eremocitrus glauca, which grows very slowly), they were inoculated with weevil larvae. Healthy $D$. abbreviatus larvae $\approx 1$ month old, weighing $18-38 \mathrm{mg}$, were selected from a colony reared on artificial diet maintained at the U.S. Horticultural Research Laboratory in Orlando. Ten larvae were inserted into the soil in each pot in a ring around the trunk of the plant, halfway between trunk and pot edge, and halfway between the soil surface and bottom (8-cm depth). Seven plants of each genotype were infested with larvae (except $F$. hindsii, where three were used) and seven were left as controls. Test plants were maintained in a greenhouse as described above for 5-6 weeks 
Table 1. Germplasm in Aurantiodeae subfamily ${ }^{\mathrm{z}}$ tested for resistance to Diaprepes abbreviatus.

\begin{tabular}{|c|c|c|c|c|}
\hline Tribe & Subtribe & Group & Species & $\operatorname{Ref}^{y}$ \\
\hline Clauseneae & Micromelinae & & None tested & --- \\
\hline Clauseneae & Clauseninae & & Murraya paniculata (L.) Jack & 4 \\
\hline Clauseneae & Clauseninae & & Glycosmis pentaphylla (Retz.) Correa & 3,4 \\
\hline Clauseneae & Merrilliinae & & None tested & --- \\
\hline Citreae & Triphasiinae & & Triphasia trifolia (Burm. f.) P. Wils. & 4 \\
\hline Citreae & Citrinae & A & Severinia buxifolia (Poir.) Tenore & 1,4 \\
\hline Citreae & Citrinae & $\mathrm{B}$ & Citropsis gilletiana Swing. \& M. Kell. & 4 \\
\hline Citreae & Citrinae & $\mathrm{C}$ & Fortunella hindsii (Champ.) Swing. & 4 \\
\hline Citreae & Citrinae & $\mathrm{C}$ & Eremocitrus glauca (Lindl.) Swing. & 4 \\
\hline Citreae & Citrinae & $\mathrm{C}$ & Poncirus trifoliata (L.) Raf. & 1 \\
\hline Citreae & Citrinae & $\mathrm{C}$ & Clymenia polyandra (Tan.) Swing. & 4 \\
\hline Citreae & Citrinae & $\mathrm{C}$ & Microcitrus australasica (F. Muell.) Swing. & 1 \\
\hline Citreae & Citrinae & $\mathrm{C}$ & Microcitrus australis (Planch.) Swing. & 4 \\
\hline Citreae & Citrinae & $\mathrm{C}$ & Citrus aurantium $\mathrm{L}$. & $1,2,4$ \\
\hline Citreae & Citrinae & $\mathrm{C}$ & Citrus hystrix DC. & 4 \\
\hline Citreae & Citrinae & $\mathrm{C}$ & Citrus limon (L.) Burm. f. & 1 \\
\hline Citreae & Citrinae & $\mathrm{C}$ & Citrus macrophylla Webster & 1,2 \\
\hline Citreae & Citrinae & $\mathrm{C}$ & Citrus reticulata Blanco & $1,2,4$ \\
\hline Citreae & Citrinae & $\mathrm{C}$ & Citrus sinensis (L.) Osbeck & 1 \\
\hline Citreae & Basamocitrinae & A & None tested & --- \\
\hline Citreae & Basamocitrinae & B & Afraegle paniculata (Schum.) Engl. & 4 \\
\hline Citreae & Basamocitrinae & B & Balsamocitrus dawei Stapf. & 4 \\
\hline Citreae & Basamocitrinae & $\mathrm{C}$ & None tested & --- \\
\hline
\end{tabular}

${ }^{2}$ Swingle (1967).

y 1 = Beavers and Hutchison (1985); 2 = Shapiro and Gottwald (1995); 3 = Shapiro et al. (1997); 4 = This manuscript.

Table 2. Common and new hybrid rootstocks tested for resistance to Diaprepes abbreviatus.

\begin{tabular}{lcc}
\hline \hline Name/Code & \multicolumn{1}{c}{ Parentage } & $\begin{array}{c}\text { Commercial } \\
\text { utilization }^{2}\end{array}$ \\
\hline Carrizo citrange & Citrus sinensis $\times$ P. trifoliata & 24 \\
Swingle citrumelo & C. paradisi Macf. $\times$ P. trifoliata & 53 \\
Smooth Flat Seville & Citrus hybrid, parentage unknown & 4 \\
Sun Chu Sha & C. reticulata & 2 \\
Sour orange & C. aurantium & 2 \\
Yuma & probably C. paradisi $\times$ P. trifoliata & 0 \\
HRS-801 & C. reticulata $\times$ P. trifoliata & 0 \\
HRS-802 & C. grandis (L.) Osbeck $\times$ P. trifoliata & 0 \\
HRS-812 & C. reticulata $\times$ P. trifoliata & 0 \\
HRS-896 & C. reticulata $\times$ P. trifoliata & 0 \\
HRS-941 & C. reticulata $\times$ P. trifoliata & 0 \\
HRS-1101 & C. reticulata $\times$ C. macrophylla & 0
\end{tabular}

${ }^{2}$ Percentage of Florida citrus nursery trees propagated on this rootstock over the last

7 years, as indicated by Florida Dept. of Agriculture (1998). to allow larvae to feed on the roots. During this initial 6 week period, D. abbreviatus larvae feed and grow most rapidly. After this feeding period, roots were washed free of soil mix and recovered larvae were counted and weighed (Shapiro et al., 1997). Root masses of both infested and control plants were severed at the soil line, air-dried, and weighed. The average root weight of seven noninfested plants of each genotype was calculated at the end of the experiment. The amount of root loss due to feeding of $D$. abbreviatus was estimated for each replicate (pot) as the difference between this mean value and the root weight for each infested plant. A comparison of infested to noninfested plants of each cultivar independently by $t$ test was used to determine resistance based on root damage because of uncontrolled factors that varied between and within experiments. Shapiro and Gottwald (1995) expressed root loss as a ratio of infested root weight : uninfested root weight. We did not use this conversion because our initial root weights varied considerably and a ratio comparison would bias resistance estimates to favor selections with larger initial root weights. Instead, resistance to root damage by larvae was assessed by a $t$ test comparison of root weight for infested and noninfested plants of each selection. Resistant types were those with no significant difference $(P \leq 0.05)$ in root dry weight between treated plants and the noninfested controls.

For comparison of means for larval growth and survival among rootstocks and species within experiments, a single factor analysis of variance (ANOVA) was used, followed by Tukey's honestly significant difference (HSD) test (SAS version 6.08, SAS Institute; and Statistica version 5.0, Statsoft, Tulsa, Okla.). All statistical comparisons used 'Carrizo' citrange as the standard because it is a commonly planted rootstock and is widely accepted as susceptible to D. abbreviatus.

For easy comparisons of cultivars/selections included in different experiments, root

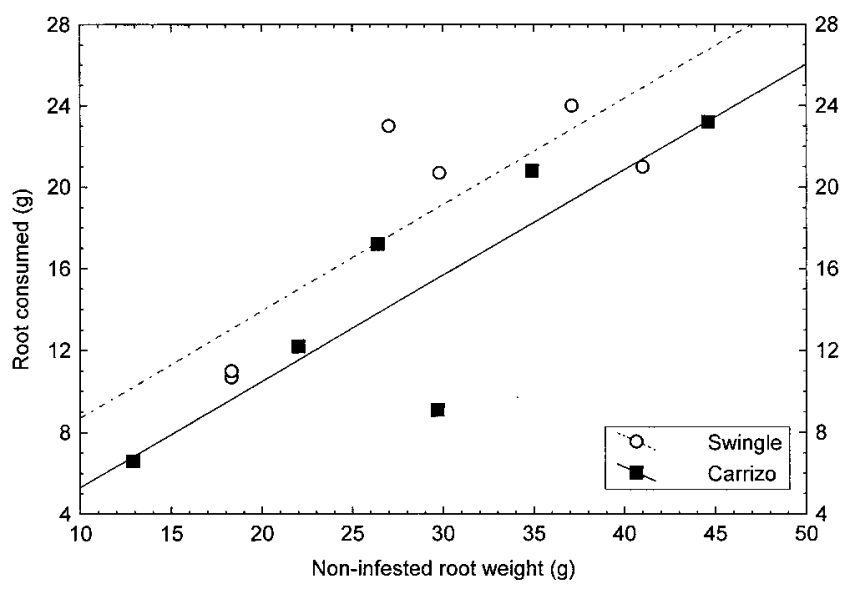

Fig. 1. Relationship of noninfested root weight to infested root consumed.

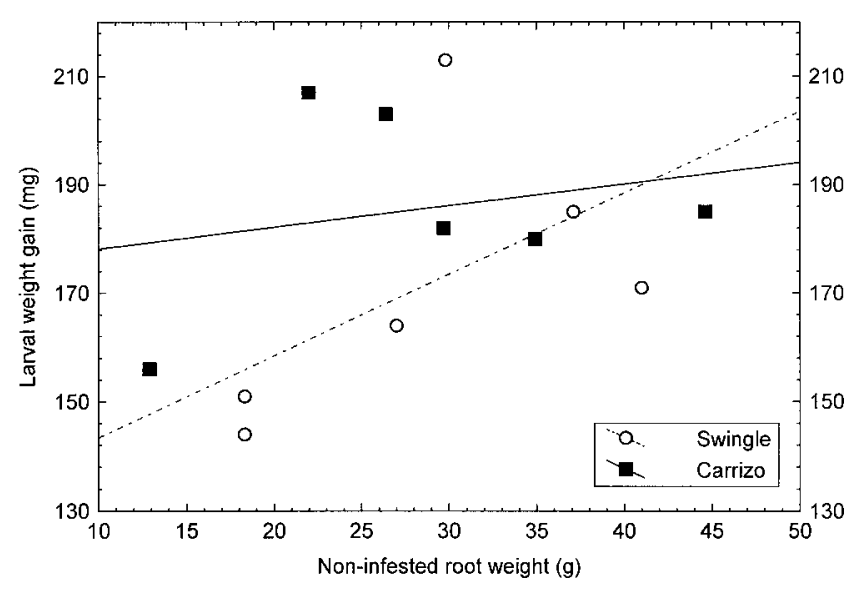

Fig. 2. Relationship of noninfested root weight to larval weight gain. 
loss and larval growth were converted to a numerical value that ranged from 1 to 4 , where 1 = highly susceptible, 2 = susceptible (by definition $=$ 'Carrizo'), $3=$ resistant, and $4=$ highly resistant. For the nine selections tested in more than one experiment, the summary rating of each of the resistance measures was expressed as an average of the values from each experiment. An overall resistance rating (average of the measures of root loss and larval growth) was also calcu- lated. This rating is proposed as an aid for quantifying levels of resistance in a preliminary study until more detailed information can be collected on resistance of individual selections.

\section{Results}

Each experiment identified one or more citrus rootstocks or citrus relatives that differed significantly from 'Carrizo' by at least
Table 3. Mean root damage to rootstocks in six experiments, means $\pm \mathrm{SE}^{2}$

\begin{tabular}{|c|c|c|c|c|}
\hline \multirow[b]{2}{*}{ Species/cultivar } & \multicolumn{2}{|c|}{ Root wt (g) } & \multirow[b]{2}{*}{$P^{\mathrm{y}}$} & \multirow[b]{2}{*}{ Rating $^{x}$} \\
\hline & Noninfested & Infested & & \\
\hline \multicolumn{5}{|c|}{ Expt. 1. Jan. 1996} \\
\hline Carrizo & $34.9 \pm 3.0$ & $14.1 \pm 1.2$ & 0.000 & $\mathrm{~S}$ \\
\hline Swingle & $18.3 \pm 1.8$ & $7.6 \pm 1.2$ & 0.000 & $\mathrm{~S}$ \\
\hline Sun Chu Sha & $47.2 \pm 5.0$ & $21.8 \pm 3.6$ & 0.001 & $\mathrm{~S}$ \\
\hline HRS-801 & $16.0 \pm 0.9$ & $8.0 \pm 0.7$ & 0.000 & $\mathrm{~S}$ \\
\hline HRS-802 & $75.2 \pm 4.6$ & $39.8 \pm 3.3$ & 0.000 & $\mathrm{~S}$ \\
\hline HRS-812 & $33.5 \pm 4.0$ & $12.4 \pm 1.5$ & 0.000 & $\mathrm{~S}$ \\
\hline HRS-896 & $30.7 \pm 3.8$ & $13.1 \pm 1.1$ & 0.001 & $\mathrm{~S}$ \\
\hline HRS-941 & $29.3 \pm 2.6$ & $10.7 \pm 1.2$ & 0.000 & $\mathrm{~S}$ \\
\hline Afraegle paniculata & $57.7 \pm 2.9$ & $30.6 \pm 4.8$ & 0.000 & $\mathrm{~S}$ \\
\hline \multicolumn{5}{|c|}{ Expt. 2. June 1996} \\
\hline Carrizo & $44.6 \pm 8.3$ & $21.3 \pm 1.6$ & 0.018 & $\mathrm{~S}$ \\
\hline Swingle & $37.1 \pm 1.7$ & $13.1 \pm 1.4$ & 0.000 & $\mathrm{~S}$ \\
\hline HRS-801 & $23.1 \pm 3.8$ & $7.1 \pm 1.5$ & 0.002 & $\mathrm{~S}$ \\
\hline HRS-802 & $39.5 \pm 6.3$ & $35.9 \pm 5.0$ & 0.655 & $\mathrm{R}$ \\
\hline HRS-896 & $24.4 \pm 2.1$ & $7.9 \pm 1.4$ & 0.000 & $\mathrm{~S}$ \\
\hline Smooth Flat Seville & $64.5 \pm 20.6$ & $52.2 \pm 6.0$ & 0.576 & $\mathrm{R}$ \\
\hline Balsamocitrus dawei & $42.0 \pm 8.6$ & $46.0 \pm 12.9$ & 0.802 & $\mathrm{R}$ \\
\hline Yuma & $55.4 \pm 4.9$ & $34.8 \pm 3.3$ & 0.005 & $\mathrm{~S}$ \\
\hline Citropsis gilletiana & $72.9 \pm 15.8$ & $39.0 \pm 5.2$ & 0.064 & $\mathrm{R}$ \\
\hline \multicolumn{5}{|c|}{ Expt. 3. July 1996} \\
\hline Carrizo & $22.0 \pm 1.5$ & $9.7 \pm 1.4$ & 0.000 & $\mathrm{~S}$ \\
\hline Swingle & $29.8 \pm 2.3$ & $9.1 \pm 1.4$ & 0.000 & $\mathrm{~S}$ \\
\hline HRS-1101 & $41.5 \pm 3.8$ & $14.5 \pm 2.3$ & 0.000 & $\mathrm{~S}$ \\
\hline Severinia buxifolia & $27.4 \pm 5.3$ & $9.8 \pm 2.3$ & 0.011 & $\mathrm{~S}$ \\
\hline HRS-941 & $32.6 \pm 3.9$ & $11.3 \pm 1.3$ & 0.000 & $\mathrm{~S}$ \\
\hline Microcitrus australis & $33.0 \pm 5.9$ & $9.7 \pm 1.5$ & 0.002 & $\mathrm{~S}$ \\
\hline Murraya paniculata & $29.3 \pm 6.6$ & $20.4 \pm 3.5$ & 0.256 & $\mathrm{R}$ \\
\hline Glycosmis pentaphylla & $37.5 \pm 5.2$ & $32.9 \pm 4.1$ & 0.501 & $\mathrm{R}$ \\
\hline Eremocitrus glauca & $15.2 \pm 1.2$ & $6.0 \pm 1.2$ & 0.000 & $\mathrm{~S}$ \\
\hline \multicolumn{5}{|c|}{ Expt. 4. Dec. 1996} \\
\hline Carrizo & $29.7 \pm 1.8$ & $20.6 \pm 3.5$ & 0.039 & $\mathrm{~S}$ \\
\hline Swingle & $41.0 \pm 3.7$ & $20.0 \pm 4.5$ & 0.003 & $\mathrm{~S}$ \\
\hline HRS-802 & $66.3 \pm 5.5$ & $44.3 \pm 5.5$ & 0.015 & $\mathrm{~S}$ \\
\hline HRS-812 & $48.7 \pm 5.1$ & $27.1 \pm 2.0$ & 0.002 & $\mathrm{~S}$ \\
\hline HRS-896 & $41.7 \pm 4.3$ & $29.5 \pm 3.2$ & 0.043 & $\mathrm{~S}$ \\
\hline Sun Chu Sha & $62.8 \pm 9.2$ & $45.8 \pm 4.4$ & 0.120 & $\mathrm{R}$ \\
\hline Triphasia trifolia & $26.4 \pm 3.4$ & $11.5 \pm 1.0$ & 0.001 & $\mathrm{~S}$ \\
\hline Clymenia polyandra & $29.2 \pm 7.0$ & $5.4 \pm 1.3$ & 0.006 & $\mathrm{~S}$ \\
\hline Citrus hystrix & $17.8 \pm 5.0$ & $5.6 \pm 1.7$ & 0.037 & $\mathrm{~S}$ \\
\hline Fortunella hindsii & $27.2 \pm 8.6$ & $4.0 \pm 0.5$ & 0.055 & $\mathrm{R}$ \\
\hline \multicolumn{5}{|c|}{ Expt. 5. Oct. 1997} \\
\hline Carrizo & $26.4 \pm 2.8$ & $9.2 \pm 1.2$ & 0.000 & $\mathrm{~S}$ \\
\hline Swingle & $27.0 \pm 3.5$ & $4.0 \pm 1.0$ & 0.000 & $\mathrm{~S}$ \\
\hline Glycosmis pentaphylla & $23.1 \pm 3.2$ & $7.7 \pm 1.0$ & 0.001 & $\mathrm{~S}$ \\
\hline \multicolumn{5}{|c|}{ Expt. 6. May 1998} \\
\hline Carrizo & $12.9 \pm 0.7$ & $6.3 \pm 0.9$ & 0.000 & $\mathrm{~S}$ \\
\hline Swingle & $18.3 \pm 1.8$ & $7.3 \pm 1.0$ & 0.000 & $\mathrm{~S}$ \\
\hline Glycosmis pentaphylla & $21.3 \pm 2.9$ & $10.4 \pm 1.2$ & 0.005 & $\mathrm{~S}$ \\
\hline HRS-802 & $26.4 \pm 1.7$ & $12.4 \pm 1.4$ & 0.000 & $\mathrm{~S}$ \\
\hline HRS-896 & $17.1 \pm 1.0$ & $9.5 \pm 1.5$ & 0.001 & $\mathrm{~S}$ \\
\hline HRS-941 & $20.8 \pm 1.8$ & $11.4 \pm 2.1$ & 0.005 & $\mathrm{~S}$ \\
\hline Citrus aurantium & $20.7 \pm 1.1$ & $4.2 \pm 0.5$ & 0.000 & $\mathrm{~S}$ \\
\hline
\end{tabular}

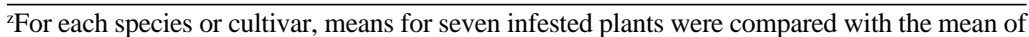
seven noninfested plants.

Significance in $t$ test.

${ }^{x}$ Ratings: $\mathrm{S}=$ susceptible; infested were significantly different from noninfested plants, $P<0.05$. $\mathrm{R}=$ resistant; infested were not significantly different from noninfested plants, $P>0.05$. one of the measures of resistance studied (Tables 3 and 4). Both highly susceptible and resistant types were identified within some individual tests, and results for genotypes included in more than one test were consistent from one test to another for most cultivars.

Shapiro and Gottwald (1995) suggested that the rootstock 'Swingle' citrumelo was somewhat more resistant to the weevil than 'Carrizo'. However, during repeated testing we found no consistent difference between the two cultivars. In particular, root loss, larval survival, and larval growth were statistically indistinguishable for the two cultivars in five of the six experiments (Tables 3 and 4). In the remaining experiment (Expt. 1), less larval growth occurred on 'Swingle' than on 'Carrizo', while root loss and larval survival were indistinguishable.

Because 'Carrizo' and 'Swingle' appeared similar in weevil resistance, data collected for the two cultivars were used to examine the relationship between root loss from infestation and noninfested root weight (Fig. 1). There was a correlation between these two parameters for both 'Carrizo' $\left(r^{2}=0.726, P<0.031\right)$ and 'Swingle' $\left(r^{2}=0.674, P<0.045\right)$.

In three of the six experiments, no significant differences in percentage root loss were observed between the cultivars/species tested. Plants of B. dawei, M. paniculata, C. gilletiana, 'Smooth Flat Seville', 'Sun Chu Sha', $G$. pentaphylla, and the hybrid HRS-802 did not suffer significant root loss from feeding of $D$. abbreviatus in at least one experiment.

The relationship between noninfested root weight and larval weight gain was nonsignificant for either 'Carrizo' $\left(r^{2}=0.056, P<\right.$ $0.651)$ or 'Swingle' $\left(r^{2}=0.319, P<0.243\right)$ (Fig. 2). We conclude that larval growth and survival were not limited by availability of food, demonstrating that our level of infestations and test duration were appropriate to minimize competition between larvae for nutritional resources. To evaluate resistance by larval growth, the larval weight gained on each species was compared with 'Carrizo' for each experiment. Several of the species supported significantly less larval growth than 'Carrizo', including B. dawei, $C$. polyandra, S. buxifolia, M. australis, $G$. pentaphylla, E. glauca, T. trifolia, C. hystrix, and hybrids HRS-801, HRS-896, and HRS941 (Table 4).

Some cultivars/species differed significantly from 'Carrizo' in larval survival in some experiments. However, larval survival appeared to follow the same patterns as larval growth, but was somewhat less sensitive than the latter measure of plant resistance. All significant differences that were observed in larval survival were mirrored by significant differences in larval growth. Therefore, larval survival was not included in the overall summary and resistance rating described below. Many of the 23 other genotypes tested during the course of the six experiments differed little from the standard, 'Carrizo', in apparent susceptibility to D. abbreviatus larvae (Tables 3 and 4). 
Table 4. Larval survival and growth on rootstocks in six experiments, means \pm SE.

\begin{tabular}{|c|c|c|c|c|}
\hline \multirow[b]{2}{*}{ Species/cultivar } & \multicolumn{2}{|c|}{ Larval survival } & \multicolumn{2}{|c|}{ Larval growth } \\
\hline & $(\%)$ & Rating & $(\mathrm{mg})$ & $\overline{\text { Rating }^{2}}$ \\
\hline \multicolumn{5}{|c|}{ Expt. 1. Jan. 1996} \\
\hline Carrizo & $91 \pm 1$ & $\mathrm{~S}$ & $180 \pm 5$ & $\mathrm{~S}$ \\
\hline Swingle & $94 \pm 2$ & $\mathrm{~S}$ & $151 \pm 6$ & $\mathrm{R}$ \\
\hline Sun Chu Sha & $94 \pm 3$ & $\mathrm{~S}$ & $173 \pm 4$ & $\mathrm{~S}$ \\
\hline HRS-801 & $76 \pm 2$ & $\mathrm{R}$ & $151 \pm 8$ & $\mathrm{R}$ \\
\hline HRS-802 & $89 \pm 5$ & $\mathrm{~S}$ & $206 \pm 4$ & $\mathrm{~S}$ \\
\hline HRS-812 & $93 \pm 4$ & $\mathrm{~S}$ & $160 \pm 7$ & $\mathrm{~S}$ \\
\hline HRS-896 & $87 \pm 4$ & $\mathrm{~S}$ & $138 \pm 8$ & HR \\
\hline HRS-941 & $89 \pm 3$ & $\mathrm{~S}$ & $166 \pm 4$ & $\mathrm{~S}$ \\
\hline Afraegle paniculata & $94 \pm 2$ & $\mathrm{~S}$ & $155 \pm 6$ & $\mathrm{~S}$ \\
\hline $\mathrm{P}(\mathrm{F}$ test $)$ & 0.003 & & 0.0001 & \\
\hline \multicolumn{5}{|c|}{ Expt. 2. June 1996} \\
\hline Carrizo & $96 \pm 3$ & $\mathrm{~S}$ & $185 \pm 8$ & $\mathrm{~S}$ \\
\hline Swingle & $89 \pm 3$ & $\mathrm{~S}$ & $185 \pm 5$ & $\mathrm{~S}$ \\
\hline HRS-801 & $77 \pm 5$ & $\mathrm{R}$ & $122 \pm 7$ & HR \\
\hline HRS-802 & $96 \pm 2$ & $\mathrm{~S}$ & $196 \pm 11$ & $\mathrm{~S}$ \\
\hline HRS-896 & $87 \pm 4$ & $\mathrm{~S}$ & $158 \pm 5$ & $\mathrm{~S}$ \\
\hline Smooth Flat Seville & $96 \pm 2$ & $\mathrm{~S}$ & $190 \pm 6$ & $\mathrm{~S}$ \\
\hline Balsamocitrus dawei & $81 \pm 3$ & $\mathrm{R}$ & $128 \pm 10$ & HR \\
\hline Yuma & $87 \pm 3$ & $\mathrm{~S}$ & $183 \pm 7$ & $\mathrm{~S}$ \\
\hline Citropsis gilletiana & $96 \pm 3$ & $\mathrm{~S}$ & $258 \pm 12$ & HS \\
\hline$P($ F test $)$ & 0.0001 & & 0.0001 & \\
\hline \multicolumn{5}{|c|}{ Expt. 3. July 1996} \\
\hline Carrizo & $83 \pm 5$ & $\mathrm{~S}$ & $207 \pm 11$ & $\mathrm{~S}$ \\
\hline Swingle & $86 \pm 5$ & $\mathrm{~S}$ & $213 \pm 20$ & $\mathrm{~S}$ \\
\hline HRS-1101 & $79 \pm 3$ & $\mathrm{~S}$ & $176 \pm 8$ & $\mathrm{~S}$ \\
\hline Severinia buxifolia & $70 \pm 7$ & $\mathrm{~S}$ & $104 \pm 11$ & HR \\
\hline HRS-941 & $70 \pm 4$ & $\mathrm{~S}$ & $145 \pm 9$ & $\mathrm{R}$ \\
\hline Microcitrus australis & $63 \pm 7$ & $\mathrm{~S}$ & $80 \pm 6$ & HR \\
\hline Murraya paniculata & $87 \pm 4$ & $\mathrm{~S}$ & $164 \pm 12$ & $\mathrm{~S}$ \\
\hline Glycosmis pentaphylla & $57 \pm 5$ & $\mathrm{~S}$ & $38 \pm 5$ & HR \\
\hline Eremocitrus glauca & $51 \pm 8$ & $\mathrm{R}$ & $81 \pm 8$ & HR \\
\hline$P($ F test $)$ & 0.0001 & & 0.0001 & \\
\hline \multicolumn{5}{|c|}{ Expt. 4. Dec. 1996} \\
\hline Carrizo & $89 \pm 3$ & $\mathrm{~S}$ & $182 \pm 10$ & $\mathrm{~S}$ \\
\hline Swingle & $87 \pm 4$ & $\mathrm{~S}$ & $171 \pm 10$ & $\mathrm{~S}$ \\
\hline HRS-802 & $93 \pm 3$ & $\mathrm{~S}$ & $228 \pm 5$ & HS \\
\hline HRS-812 & $91 \pm 1$ & $\mathrm{~S}$ & $199 \pm 9$ & $\mathrm{~S}$ \\
\hline HRS-896 & $87 \pm 5$ & $\mathrm{~S}$ & $158 \pm 12$ & $\mathrm{~S}$ \\
\hline Sun Chu Sha & $94 \pm 2$ & $\mathrm{~S}$ & $209 \pm 10$ & $\mathrm{~S}$ \\
\hline Triphasia trifolia & $56 \pm 6$ & HR & $55 \pm 4$ & HR \\
\hline Clymenia polyandra & $89 \pm 4$ & $\mathrm{~S}$ & $124 \pm 5$ & $\mathrm{R}$ \\
\hline Citrus hystrix & $83 \pm 2$ & $\mathrm{~S}$ & $89 \pm 12$ & HR \\
\hline Fortunella hindsii & $73 \pm 3$ & $\mathrm{~S}$ & $150 \pm 13$ & $\mathrm{~S}$ \\
\hline$P($ F test $)$ & 0.0001 & & 0.0001 & \\
\hline \multicolumn{5}{|c|}{ Expt. 5. Oct. 1997} \\
\hline Carrizo & $86 \pm 4$ & $\mathrm{~S}$ & $203 \pm 14$ & $\mathrm{~S}$ \\
\hline Swingle & $81 \pm 3$ & $\mathrm{~S}$ & $164 \pm 17$ & $\mathrm{~S}$ \\
\hline Glycosmis pentaphylla & $67 \pm 7$ & $\mathrm{R}$ & $30 \pm 5$ & HR \\
\hline$P($ F test $)$ & 0.044 & & 0.0001 & \\
\hline \multicolumn{5}{|c|}{ Expt. 6. May 1998} \\
\hline Carrizo & $81 \pm 7$ & $\mathrm{~S}$ & $156 \pm 9$ & $\mathrm{~S}$ \\
\hline Swingle & $90 \pm 4$ & $\mathrm{~S}$ & $144 \pm 6$ & $\mathrm{~S}$ \\
\hline Glycosmis pentaphylla & $61 \pm 7$ & $\mathrm{~S}$ & $35 \pm 3$ & HR \\
\hline HRS-802 & $74 \pm 6$ & $\mathrm{~S}$ & $214 \pm 5$ & HS \\
\hline HRS-896 & $79 \pm 6$ & $\mathrm{~S}$ & $116 \pm 9$ & $\mathrm{R}$ \\
\hline HRS-941 & $80 \pm 4$ & $\mathrm{~S}$ & $145 \pm 9$ & $\mathrm{~S}$ \\
\hline Citrus aurantium & $90 \pm 3$ & $\mathrm{~S}$ & $195 \pm 8$ & HS \\
\hline$P($ F test $)$ & 0.012 & & 0.0000 & \\
\hline
\end{tabular}

${ }^{2}$ Ratings: Carrizo is susceptible (S). All means rated as $\mathrm{S}$ were not distinguished from 'Carrizo' by Tukey's honestly significant difference (HSD) test at $P \leq 0.05$ and are also considered susceptible. HS = highly susceptible, more larval survival or growth than 'Carrizo', and distinguished from it by Tukey's HSD test at $P \leq 0.05 . \mathrm{R}=$ resistant, less larval survival or growth than 'Carrizo', and distinguished from it by Tukey's HSD test at $P \leq 0.05$. $\mathrm{HR}=$ highly resistant, less larval survival or growth than 'Carrizo', and distinguished from it by Tukey's HSD test at $P \leq 0.001$.
Based on these experiments, the species Balsamocitrus dawei has a high level of resistance to D. abbreviatus larvae, suffering little root damage and inhibiting larval growth, while G. pentaphylla, E. glauca, M. australis, S. buxifolia, T. trifolia, and $C$. hystrix have a lesser level of resistance that was evidenced mainly by a depression in larval growth (Table 5). The cuttings of $C$. hystrix used in testing appeared healthy and similar to seedlings. However, it should be noted that if seedlings had been used for this cultivar, rather than cuttings, there would have been more genetic variability among the plants tested and differences in root structure might have affected test results. All of the commercially used rootstocks that we tested in these experiments ('Swingle', 'Smooth Flat Seville', 'Sun Chu Sha', and $C$. aurantium), many new hybrid rootstocks, and some of the related species were as susceptible to D. abbreviatus weevil larvae as was 'Carrizo'. One new hybrid rootstock, HRS801 , depressed the growth of $D$. abbreviatus larvae consistently in two experiments. Only C. aurantium was possibly more susceptible to Diaprepes than 'Carrizo', based on the resistance index.

\section{Discussion}

This series of experiments identified several sources of resistance to be used in future research and breeding efforts. Information in the literature on sexual and graft compatibility of citrus relatives is limited (Swingle, 1967). However, the species most resistant to Diaprepes weevil larvae, Balsamocitrus dawei, is considered sexually and graft incompatible with the genus Citrus, eliminating the opportunity to transmit the resistance factors by sexual hybridization or to use this species directly as a rootstock. The species $G$. pentaphylla is also sexually incompatible with citrus, but is partially graft-compatible with some citrus species (Bowman, unpublished data). A similar situation may exist with $T$. trifolia. Experiments are underway to identify suitable interstocks to allow use of $G$. pentaphylla rootstocks with commercial scions in areas infested by $D$. abbreviatus. The biochemical basis of resistance in $G$. pentaphylla has been investigated (Shapiro et al., 1997), and one component of this resistance has been identified as dehydrothalebanin (Shapiro et al., in press). This information might permit manipulation of biochemical pathways in existing citrus rootstocks, via genetic transformation, to induce "Glycosmis-type" resistance.

In contrast, antibiosis from $S$. buxifolia, E. glauca, M. australis, and C. hystrix could potentially be introgressed via breeding into citrus rootstocks because these four species are sexually compatible (Swingle, 1967; Medina-Filho et al., 1998) with some common species of the genus Citrus. Although resistance to $D$. abbreviatus was not a factor in their creation, hundreds of sexual hybrids between Citrus species and Eremocitrus or Microcitrus already exist in the germplasm of the Ft. Pierce USDA breeding programs. 
Table 5. Resistance of citrus rootstocks and species to D. abbreviatus over six experiments.

\begin{tabular}{|c|c|c|c|c|}
\hline Species/cultivar & Expts. & $\begin{array}{c}\text { Root loss } \\
\text { (R1) }\end{array}$ & $\begin{array}{c}\text { Larval growth } \\
\text { (R2) }\end{array}$ & $\begin{array}{c}\text { Resistance rating }^{2} \\
(\mathrm{R} 1+\mathrm{R} 2) \div 2\end{array}$ \\
\hline Balsamocitrus dawei & 2 & 3 & 4 & 3.5 \\
\hline Glycosmis pentaphylla & $3,5,6$ & 2.3 & 4 & 3.2 \\
\hline Eremocitrus glauca & 3 & 2 & 4 & 3 \\
\hline Microcitrus australis & 3 & 2 & 4 & 3 \\
\hline Severinia buxifolia & 3 & 2 & 4 & 3 \\
\hline Triphasia trifolia & 4 & 2 & 4 & 3 \\
\hline Citrus hystrix & 4 & 2 & 4 & 3 \\
\hline HRS-801 & 1,2 & 2 & 3.5 & 2.8 \\
\hline Murraya paniculata & 3 & 3 & 2 & 2.5 \\
\hline Clymenia polyandra & 4 & 2 & 3 & 2.5 \\
\hline Smooth Flat Seville & 2 & 3 & 2 & 2.5 \\
\hline Sun Chu Sha & 1,4 & 2.5 & 2 & 2.3 \\
\hline Hybrid HRS-896 & $1,2,4,6$ & 2 & 2.5 & 2.3 \\
\hline Hybrid HRS-941 & $1,3,6$ & 2 & 2.3 & 2.2 \\
\hline Swingle & $1,2,3,4,5,6$ & 2 & 2.2 & 2.1 \\
\hline Carrizo & $1,2,3,4,5,6$ & 2 & 2 & 2 \\
\hline Yuma & 2 & 2 & 2 & 2 \\
\hline HRS-812 & 1,4 & 2 & 2 & 2 \\
\hline HRS-1101 & 3 & 2 & 2 & 2 \\
\hline Afraegle paniculata & 1 & 2 & 2 & 2 \\
\hline Citropsis gilletiana & 2 & 3 & 1 & 2 \\
\hline Fortunella hindsii & 4 & 2 & 2 & 2 \\
\hline HRS-802 & $1,2,4,6$ & 2.3 & 1.5 & 1.9 \\
\hline Citrus aurantium & 6 & 2 & 1 & 1.5 \\
\hline
\end{tabular}

${ }^{2}$ Ratings: 1 = highly susceptible; 2 = susceptible; 3 = resistant; 4 = highly resistant.

Somatic hybrids between Citrus species and Microcitrus or Severinia have also been produced and are under evaluation (Grosser et al., 1996). Caution is still necessary in using these sources of resistance because we do not know how the short-term depression in larval growth that occurred from feeding on these four species relates to weevil maturation, reproduction, or population dynamics under field conditions. These species all suffered significant root damage from $D$. abbreviatus during the greenhouse testing.

The observation that hybrid HRS-801 significantly reduced larval growth and survival may indicate that a modest level of resistance occurs in some hybrids of Citrus reticulata $\mathrm{X}$ Poncirus trifoliata. This type of hybrid also has potential to combine other useful rootstock traits, including tree size control and resistance to citrus tristeza virus, citrus nematode, phytophthora diseases, cold, and citrus blight (Bowman, 1998; Wutscher and Bowman, 1999; Wutscher and Hill, 1995). However, we cannot eliminate the possibility that reduction in larval health for this selection may have been the result of a relatively small root mass $(<25 \mathrm{~g})$ for the tests with this selection, despite the conclusion that root weight (in the range of 25-45 g) was not associated with larval growth (Fig. 2). As with the antibiosis of E. glauca, M. australis, and S. buxifolia, and C. hystrix, better definition is needed for how this short-term depression in larval growth relates to longer-term resistance issues.

For future greenhouse testing, use of potting mix without perlite, as was done in
Expt. 6, may reduce the difficulty in recovering weevil larvae at the conclusion of tests. It did not appear to significantly affect testing of either standard susceptible cultivars like 'Carrizo' and 'Swingle' or the resistant species G. pentaphylla. Standardization of initial root mass of selections to be tested is important to minimize the potential influence of this factor on root damage and larval growth. Glycosmis pentaphylla appeared to be a good resistant control to include in future greenhouse tests for Diaprepes resistance; B. dawei may also be used for this purpose, but is probably less convenient because of a long juvenile period, greater seedling variability, and very limited seed sources in the United States.

The research described here has identified several new potential sources of resistance to Diaprepes weevil larvae, defined relative level of susceptibility for several common and new rootstock selections, and identified ways to improve greenhouse testing for weevil resistance. There appear to be several long-term approaches available for future development of new rootstocks resistant to Diaprepes weevil.

\section{Literature Cited}

Abrahamson, S.G. 1994. Attacking citrus root weevil, p. 10, 12, 15. In: Frank C. Garner (ed.). Florida Grower and Rancher.

Beavers, J.B., R.E. Woodruff, S.A. Lovestrand, and W.J. Schroeder. 1979. Bibliography of the sugarcane rootstock borer weevil, Diaprepes abbreviatus. Bul. Entomol. Soc. Amer. 25:2529.
Beavers, J.B. and D.J. Hutchison. 1985. Evaluation of selected Citrus spp. and relatives for suceptibility to root injury by Diaprepes abbreviatus larvae (Coleoptera: Curculionidae). Fla. Entomol. 68(1):222-223.

Bowman, K.D. 1998. Performance of 'Fallglo' citrus hybrid on ten rootstocks in Lake County. Proc. Fla. State Hort. Soc. 111:177-180.

Florida Dept. of Agriculture. 1998. Annual report: Bureau of citrus budwood registration. Div. Plant Industry, Winter Haven, Fla.

Grosser, J.W. and C.W. McCoy. 1996. Feeding response of first instar larvae of Diaprepes abbreviatus to different novel intergeneric citrus somatic hybrids. Proc. Fla. State Hort. Soc. 109:62-66.

Grosser, J.W., F.A.A. Mourao-Fo, F. G. Gmitter, Jr., E.S. Louzada, J. Jiang, K. Baergen, A. Quiros, C. Cabasson, J.L. Schell, and J.L. Chandler. 1996. Allotetraploid hybrids between citrus and seven related genera produced by somatic hybridization. Theor. Appl. Genet. 92:577-582.

McCoy, C.W., L.W. Duncan, and E. D. Quintela. 1996. A review of IPM strategies for citrus root weevils with emphasis on microbial control. Proc. Intl. Soc. Citricult. 1996, Vol. 1:638641.

Medina-Filho, H.P., R. Bordignon, and R.M.L. Ballve. 1998. Sunkifolias and Buxisunkis: Sexually obtained reciprocal hybrids of Citrus sunki X Severinia buxifolia. Genet. Mol. Biol. 21:129-133

Norman, P.A., A.G. Selhime, and R.A. Sutton. 1974. Feeding damage to five citrus rootstocks by larvae of Diaprepes abbreviatus (Coleoptera: Curculionidae). Fla. Entomol. 57:296.

Schroeder, W.J. 1988. Entomogenous nematodes for root weevil control in citrus, p. 1223-1226. In: R. Goren and K. Mendel (eds.), Proc. Sixth Intl. Citrus Congr., Tel Aviv, Israel, 6-11 Mar., 1988. Balaban, Philadelphia.

Schroeder, W.J. and J.B. Beavers. 1977. Citrus root weevils in Florida: identification, biology, and control. Proc. Intl. Soc. Citricult. 2:498500.

Shapiro, J.P., K.D. Bowman, and S.L. Lapointe. 2000. Dehydrothalebanin, a source of resistance from Glycosmis pentaphylla against the citrus root weevil Diaprepes abbreviatus. J. Agr. Food Chem. 48:4404-4409.

Shapiro, J.P., K.D. Bowman, and H. Smith. 1997. Resistance of citrus rootstocks and Glycosmis pentaphylla against larval citrus root weevils, Diaprepes abbreviatus, in live root or dietincorporation assays. Fla. Entomol. 80:471477.

Shapiro, J.P. and T.R. Gottwald. 1995. Resistance of eight cultivars of citrus rootstock to a larval root weevil, Diaprepes abbreviatus L. (Coleoptera: Curculionidae). J. Econ. Entomol. 88:148-154.

Swingle, W.T. and P.C. Reece. 1967. The botany of citrus and its wild relatives. p. 190-430. In: W. Reuther, H.J. Webber, W.D. Batchelor (eds.). The citrus industry. Vol. 1. Univ. of Calif. Press, Berkeley.

Wutscher, H.K. and K.D. Bowman. 1999. Performance of 'Valencia' orange on 21 rootstocks. HortScience 34:622-624.

Wutscher, H.K. and L.L. Hill. 1995. Performance of 'Hamlin' orange on 16 rootstocks in eastcentral Florida. HortScience 30:41-43. 